\title{
Antidiabetic Treatments for Diabetes with Liver Cirrhosis
}

ISSN: 2578-0263

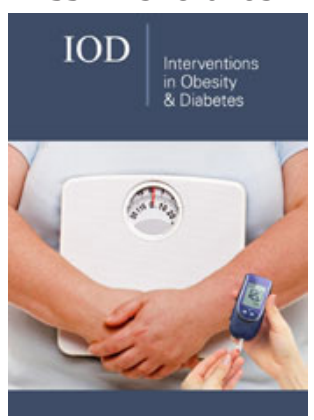

*Corresponding author: Yoshio Sumida, Division of Hepatology and Pancreatology, Department of Internal Medicine, Aichi Medical University, Japan

Submission: 監 February 03, 2020

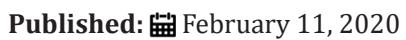

Volume 3 - Issue 4

How to cite this article: Yoshio Sumida, Masashi Yoneda, Katsutoshi Tokushige, Miwa Kawanaka, Hideki Fujii, et al. Antidiabetic Treatments for Diabetes with Liver Cirrhosis. Interventions Obes Diabetes 3(4). IOD.000566.2020.

DOI: $10.31031 /$ IOD.2020.03.000566

Copyright@ Yoshio Sumida. This article is distributed under the terms of the Creative Commons Attribution 4.0 International License, which permits unrestricted use and redistribution provided that the original author and source are credited.
Yoshio Sumida ${ }^{1 *}$, Masashi Yoneda ${ }^{1}$, Katsutoshi Tokushige ${ }^{2}$, Miwa Kawanaka ${ }^{3}$, Hideki Fujiii ${ }^{4}$, Masato Yoneda ${ }^{5}$, Kento Imajo ${ }^{5}$, Hirokazu Takahashi' ${ }^{6}$, Yuichiro Eguchi $^{6}$, Masafumi Ono ${ }^{7}$, Yuichi Nozaki ${ }^{8}$, Hideyuki Hyogo ${ }^{9}$, Masahiro Koseki ${ }^{10}$, Yuichi Yoshida $^{11}$, Takumi Kawaguchi ${ }^{12}$, Yoshihiro Kamada ${ }^{13}$, Takeshi Okanoue ${ }^{14}$ and Atsushi Nakajima ${ }^{5}$, Japan Study Group of NAFLD (JSG-NAFLD)

${ }^{1}$ Division of Hepatology and Pancreatology, Department of Internal Medicine, Aichi Medical University, Japan

${ }^{2}$ Department of Internal Medicine, Institute of Gastroenterology, Tokyo Women's Medical University, Tokyo, Japan.

${ }^{3}$ Department of General Internal Medicine, Kawasaki Medical School, Japan.

${ }^{4}$ Department of Hepatology, Graduate School of Medicine, Osaka City University, Osaka, Japan ${ }^{5}$ Division of Gastroenterology, Yokohama City University Graduate School of Medicine, Yokohama, Japan

${ }^{6}$ Department of Internal Medicine, Saga Medical School, Saga University, Saga, Japan

${ }^{7}$ Division of Gastroenterology and Hepatology, Department of Internal Medicine, Tokyo Women's Medical University Medical Center East, Tokyo, Japan.

${ }^{8}$ Department of Gastroenterology, National Center for Global Health and Medicine, Tokyo, Japan.

${ }^{9}$ Department of Gastroenterology, JA Hiroshima Kouseiren General Hospital, Japan

${ }^{10}$ Department of Cardiovascular Medicine, Osaka University Graduate School of Medicine, Japan

${ }^{11}$ Department of Gastroenterology, Suita City Hospital, Osaka, Japan

${ }^{12}$ Division of Gastroenterology, Department of Medicine, Kurume University School of Medicine, Kurume, Japan

${ }^{13}$ Department of Molecular Biochemistry \& Clinical Investigation, Osaka University Graduate School of Medicine, Suita, Osaka, Japan.

${ }^{14}$ Hepatology Center, Saiseikai Suita Hospital, Japan

\section{Abstract}

Diabetes is associated with about $30 \%$ of liver cirrhosis and affects prognosis, carcinogenesis, and the onset of complications. In cirrhosis, fasting normal and postprandial hyperglycemia are often present, and evaluation by glucose tolerance test or continuous blood glucose measurement is also considered. Although no treatment strategy has been established, if renal function is maintained, metformin will be the first choice, but for renal dysfunction cases and metformin ineffective cases, insulin will be indicated. Although the efficacy and safety of incretin-related drugs and sodium-glucose cotransporter 2 inhibitors have not been established, they can be expected to be effective in cases with obesity. On the viewpoint of preventing sarcopenia and hepatocarcinogenesis, antidiabetic drugs should be selected. Exogenous insulin should be indicated for patients with Child $\mathrm{C}$ grade. This review discusses antidiabetic treatments for diabetes with liver cirrhosis.

Keywords: Metformin; Insulin; Incretin; Sodium-glucose cotransporter 2; Glucagon like peptide 1

Abbreviations: GA: Glycated Albumin; CLD: Chronic Liver Disease; HCC: Hepatocellular Carcinoma; CGMS: Continuous Glucose Monitoring System; FGM: Flash Glucose Monitoring; NASH: Nonalcoholic Steatohepatitis; $\alpha$ GI: Alpha Glucosidase Inhibitor; SGLT2: Sodium-Glucose Cotransporter 2; DPP-4: Dipeptidyl Peptidase-4

\section{Introduction}

In cirrhosis, diabetes is complicated by $30 \%$ and glucose intolerance by $80 \%$ due to decrease in glucose processing ability in liver and skeletal muscle [1], decrease in insulin clearance and hyperinsulinemia due to portal circulation shunt. In addition, hypoglycemia at 
night and in the early morning is likely to occur because of decreased glycogen storage in the liver and inadequate gluconeogenesis. These glucose metabolism disorders have an adverse effect on the prognosis and risk of liver carcinogenesis, so their diagnosis and treatment are important issues. Glycated hemoglobin (HbA1c), which is usually used for diagnosis and follow-up of diabetes, is not reliable as an index of glycemic control in cirrhotic patients because it shows false low value by hemolysis due to hypersplenism, and it is better to use glycated albumin (GA) as an index. However, in decompensated liver cirrhosis, the ability to synthesize albumin is significantly reduced and may affect GA, so caution is required to evaluate glycemic control in patients with cirrhosis. It has also been suggested that the average value of HbA1c plus GA/3 should be used as chronic liver disease (CLD)-HbA1c from within Japan [2]. Serum fructosamine may be more suitable test for monitoring blood glucose levels [3]. As described above, in patients with CLD, measurement of fasting blood glucose alone may miss diabetes, and postprandial blood glucose measurement and glucose tolerance test should be considered. On the other hand, there is also evidence that diabetes promotes liver fibrosis and increases liver carcinogenesis, and liver fibrosis and diabetes are closely related.

\section{Practical Treatment}

\section{Life-style modification}

According to the guidelines on nutritional management in Japanese patients with liver cirrhosis aimed at preventing hepatocellular carcinoma (HCC), the standard for dietary intake is $25 \mathrm{kcal} / \mathrm{kg}$ (ideal body weight) per day, with protein intake of $1.0-1.5 \mathrm{~g} / \mathrm{kg} /$ day and a $20-25 \%$ lipid: energy ratio [4].Taking snacks at bedtime (split meals) is effective for nighttime and early morning hypoglycemia measures. When liver cirrhosis progresses, various complications appear, and administration of a nutrient for liver failure is necessary to prevent salt restriction (5 to $7 \mathrm{~g}$ ) for the appearance of ascites fluid, hypoalbuminemia and hepatic encephalopathy. However, it is necessary to be careful because a nutrient for liver failure may lead to excessive intake of calories and may lead to worsening of diabetes [5]. Exercise therapy should be recommended if you do not have significant ascites or encephalopathy to increase muscle mass.

\section{How to make treatment strategy}

The use of antidiabetic drugs in patients with cirrhosis is debated $[1,6,7]$ because adverse events may be more common and treatment targets may be different from diabetic patients without cirrhosis. Otherwise, the presence of T2DM in liver cirrhosis increases the risk of liver and not liver-related complications and death, so pharmacological approaches to this clinical setting should be safe and should improve survival. In the selection of treatment strategy, drugs are selected by comprehensively judging the evaluation of renal function, presence or absence of combined disease such as ascites, age, prognosis of liver disease. In the past, it was not easy to know blood glucose fluctuation at night, but in recent years continuous glucose monitoring system (CGMS) [8] or flash glucose monitoring (FGM) have become available, and early detection of glucose intolerance in patients with liver cirrhosis, selection of appropriate therapeutic agents. There is a high possibility of contributing significantly to dose setting and hypoglycemia control, and improvement in prognosis is also expected. There is no evidence for the target values of HbA1c or GA in the treatment of diabetes in patients with liver cirrhosis, and it is not clear to what extent strict blood glucose control should be aimed.

\section{How to select pharmacotherapies (Table 1)}

Table 1: Antidiabetic drug for patients with cirrhosis.

\begin{tabular}{|c|c|c|c|c|c|c|c|}
\hline Drugs & $\begin{array}{c}\text { Cardioprotective } \\
\text { Effect }\end{array}$ & $\begin{array}{l}\text { Reno Protective } \\
\text { Effects }\end{array}$ & $\begin{array}{l}\text { Hepatocarci-no- } \\
\text { genesis }\end{array}$ & Muscle Volume & $\begin{array}{l}\text { Adverse Effects/ } \\
\text { Concerns }\end{array}$ & Child B & Child C \\
\hline SU & $?$ & $?$ & $\uparrow$ & $\downarrow$ & Hypoglycemia & $\begin{array}{l}\text { Low dose } \\
\text { preferred }\end{array}$ & Avoided \\
\hline $\begin{array}{l}\text { Exogenous } \\
\text { insulin }\end{array}$ & $?$ & $?$ & $\uparrow$ & $\uparrow / ?$ & Hypoglycemia & Indicated & Indicated \\
\hline Metformin & ० & ० & $\begin{array}{c}\downarrow \\
\uparrow(\text { sorafenib })\end{array}$ & $?$ & Lactic acidosis & $<1500 \mathrm{mg} / \mathrm{d}$ & Avoided \\
\hline Pioglitazone & $?$ & $?$ & $?$ & $?$ & $\begin{array}{c}\text { Edema Heart } \\
\text { failure Osteopenia } \\
\text { (women) }\end{array}$ & Avoided & Avoided \\
\hline$\alpha \mathrm{GI}$ & $?$ & $?$ & $?$ & $?$ & Poor adherence & Safe & Not preferred \\
\hline DDP-4 inhibitor & $\triangle$ & $\circ$ & $\uparrow ?$ & $\rightarrow$ & $\begin{array}{l}\text { IBD? Cholangiocar- } \\
\text { cinoma? }\end{array}$ & With caution & Not preferred \\
\hline GLP-1RA & ○ & ○ & $?$ & $\rightarrow$ or $\uparrow$ & GI symptoms & Avoided & Avoided \\
\hline SGLT2 inhibitor & () & (-) & $?$ & $\downarrow$ ? & $\begin{array}{l}\text { Urogenital infec- } \\
\text { tion Dehydrosis }\end{array}$ & With caution & Avoided \\
\hline
\end{tabular}

SU: Sulphonyl Urea; aGI: a Glucosidase Inhibitor; DDP-4; Dipeptidyl Peptidase-4; sodium-glucose cotransporter 2; GI: Gastrointestinal; IBD: Inflammatory Bowel Disease; GLP-1RA: Glucagon Like Peptide-1 Receptor Agonist. 
Metformin: Metformin is specified as the first choice in the American Diabetes Association (ADA) due to its usefulness and medical economic benefits [9]. In the past, liver cirrhosis had a high risk of lactic acidosis and was considered to be a contraindication. However, a large cohort study showed that the continuation of metformin use after cirrhosis diagnosis significantly improved survival in all stages of cirrhosis, suggesting that metformin is safe and well tolerated also in patients with decompensated liver disease [10]. Recent studies have revealed that the frequency of lactic acidosis is not high if renal function is maintained, and metformin even in cases with liver cirrhosis is the first choice of medication. Particularly, it was shown that metformin had a protective effect only in patients with NASH-related cirrhosis, probably due to the pleiotropic effects of metformin in cell proliferation and differentiation, in apoptosis and inflammation and in metabolic pathways of glucose and lipid homeostasis [11]. In particular, the effect on prognosis that has been announced recently has a variety of useful data such as a $57 \%$ decrease in the mortality rate of cirrhosis [12], an encephalopathy suppressive effect, and a liver carcinogenic suppressive effect. However, it should be not used in patients with Child-Pugh class $\mathrm{C}$ cirrhosis and in presence of severe renal impairment for the risk of lactic acidosis [7].

Thiozolidine: The thiozolidine derivative improves insulin resistance through PPAR $\gamma$ activation. Troglitazone and rosiglitadine have been discontinued due to serious problems such as liver injury and heart failure. Although the efficacy of pioglitazone currently on the market has been established for nonalcoholic steatohepatitis (NASH) [13-15], the efficacy and safety for NASH-related cirrhosis have not been established. Pioglitazone is difficult to use due to concerns such as edema, weight gain and carcinogenesis. It should be avoided in patients whose liver enzymes are $>3$ times ULN range. Pioglitazone may be used in Child-Pugh Class A patients. However, it should be avoided in Class B and C patients [7]. The development of novel thiozolidine derivatives targeted to mitochondria (mTOT) is in progress [16], and we hope to overcome these side effects.

Sulfonyl urea/glinide: There is a risk of hypoglycemia, and administration to patients with cirrhosis is not recommended in principle [17]. If used, lower doses of sulfonyl urea should be preferred in Child-Pugh Class A and B. SUs should be avoided in patients with Child-Pugh Class C [7]. In alcoholic liver cirrhosis in particular, pancreatic $\beta$ cells may be exhausted and not recommended. Although glinide may be used in Child-Pugh Class A patients, it is not preferred in Child-Pugh Class B and C patients [7].

Alpha glucosidase inhibitor: It has been reported that alpha glucosidase inhibitor $(\alpha \mathrm{GI})$ significantly improve post-prandial hyperglycemia in a double-blind comparative study of 100 patients with compensated cirrhosis [18]. In addition to improving blood sugar, it has also been reported that when the cirrhotic patient with grade $1 / 2$ encephalopathy is treated with acarbose $300 \mathrm{mg}$ for 8 weeks, ammonia in the blood is reduced by increasing the number of stools [19]. $\alpha$ GI have improved blood sugar and ammonia levels compared to placebo in patients with liver cirrhosis and diabetes mellitus treated with insulin [20]. Especially for postprandial hyperglycemic cases, there are reports of side effects such as elevation of ALT, and attention is given to liver function. Also, compliance with medication compliance is key because it is administered three times a day. $\alpha$ GI are safe in Child-Pugh Class A and B. However, they are not preferred in Class C patients $[1,7]$.

Incretin related drugs: It is speculated that dipeptidyl peptidase-4 (DPP-4) inhibitors can be safely used in liver cirrhosis in a pharmacokinetic manner [21], and it has been reported that DPP-4 inhibitors suppress liver fibrosis in animal models [22]. However, there is no large-scale data for DPP4 inhibitors in patients with liver cirrhosis and diabetes [1], and the same is true based on the fact that the cardiovascular event suppression effect is poor and the recent risk of developing inflammatory bowel disease is increased by about $75 \%$ [23]. I would like to expect even incretin related drugs rather to glucagon like peptide- 1 receptor agonist (GLP-1RA). Although the efficacy of GLP-1 RA for cirrhosis is unknown, it can be the first choice, especially for NASH cirrhosis, based on results such as cardiorenal protection (LEADER trial, AWARD10 study, and SUSUATIN 6 study). Because the preparation is an injection preparation, once a week the preparation has high compliance and patient satisfaction, and dulaglutide and semaglutide are recommended. Semaglutide is currently in clinical trials for NASH (SEMA-NASH study), but cirrhosis (stage 4) cases have not been entered. Dulaglutide's disposable syringes are easy for nonspecialists to use. Semaglutide is highly effective in reducing weight and improving blood sugar but has just been approved and has little experience with it. In addition, clinical trials for NASH are currently underway, but cirrhosis is not included. Drugs of this class can be administered to Child-Pugh Class A patients. However, due to scarcity of data in hepatic impairment patients, GLP-1RAs should be avoided in Class B and C patients [7].

Sodium-glucose cotransporter 2 (SGLT2) inhibitors: It is a drug that can be a candidate for treatment in view of body weight, body fat reduction, cardiorenal protection, etc. We also reported the ALT improvement effect in the sub-analysis of the national trial for diabetic patients [24] and the reduction effect of liver steatosis evaluated by MRI [25]. However, if liver fibrosis progresses, it may be suggested that the ALT-lowering effect may be inferior [26] and we hope for future research results. Although cases of liver cirrhosis have been reported only in a case report [27], administration to cirrhosis with sarcopenia should be exercised in consideration of the possibility of decrease in muscle mass. In addition, in cases of poor hepatic reserve, there is also concern about deterioration of encephalopathy and the like due to dehydration, so we have limited cases to around Child A; however, SGLT-2 inhibitors should be used with caution in Class B patients. Agents of this class should better be avoided in Class C patients [1,7]. On the other hand, as it has $\mathrm{Na}$ excretion function, ascites improvement effect is expected rather in the ascites merger case [28], but there is not enough 
data. Furthermore, although there is a report of suppression of carcinogenesis in animal models [29], it is not clear in clinical data, and we hope for future data. With reference to the package insert of each drug for SGLT2 inhibitor, there are many drugs that are carefully administered to "patients with severe liver dysfunction", and the following two drugs without such description are recommended in this article: canagliflozin and luseogliflozin. Care must be taken for urinary tract infections such as urinary cystitis and tinea genitalia, but it can be expected to be useful for obese cases.

Insulin: Conventionally, short-acting insulin is used in principle in diabetes complicated with liver cirrhosis [30]. It is difficult to set the dose because of insulin resistance and the reduced ability to metabolize insulin, and it is desirable to monitor blood glucose at hospitalization [31]. Guidelines from the ADA highlight the importance of insulin and suggest frequent dose adjustment and careful glucose monitoring for T2DM and CLD patients [7]. Although there is a report that cautions are necessary because hypoglycemic symptoms are less likely to occur if a beta receptor antagonist is administered orally for the prevention of hemorrhage for varicose veins [31], but there is no evidence that the frequency of hypoglycemia has increased. The combination of beta receptor antagonist and insulin is not a contraindication. In patients with Child-Pugh class $\mathrm{C}$ cirrhosis, insulin therapy remains the treatment of choice for co-existing T2DM [32].

Novel drug underdevelopment: Imeglimin [33], a novel diabetes drug currently in phase 3 clinical trials in Japan, acts on muscle, pancreatic $\beta$ cells, and liver, has mitochondrial metabolism improving activity, and sub-analysis of phase 2 clinical trials in Japan also shows improvement in ALT value. Obechicholic acid, an farnesoid $\mathrm{X}$ receptor activator, is currently undergoing a global clinical trial for NASH fibrosis (REGENERATE trial) and NASH cirrhosis (REVERSE trial), but its insulin resistance ameliorating effect has also been shown [34] and we hope to see the efficacy of cirrhosis in the future. Proteomic profiles that include plasma Sirt 1 and protein analysis are critical to determine defects in the nuclear-mitochondria interaction relevant to the severity of NAFLD or diabetes [35].

\section{Antidiabetic Medications on the Viewpoint of Sarcope- nia}

Sarcopenia is a prevalent muscle abnormality in patients with cirrhosis that is associated with higher mortality pre- or postliver transplantation, a higher rate of infection and longer hospital stay, hepatic encephalopathy, poor quality of life, and increased healthcare cost [36]. We should select antidiabetic medications in cirrhotic patients on the viewpoint of sarcopenia [37,38], but for the majority, their effects on sarcopenia components are unclear. Metformin increases AMP-activated protein kinase activity, which inhibits the mechanistic target of rapamycin (mTOR), a key factor in muscle growth. However, metformin is being investigated as an adjunct therapy to resistance training in older adults, as it may assist in the mobilization of M2 macrophages, which could have anti-inflammatory properties in skeletal muscle. In the MrOS study, men with diabetes treated with metformin alone and metformin plus thiazolidinediones (insulin sensitizers) over $3.5 \pm 0.7$ years follow-up had an attenuated loss of total lean mass and ALM compared to men with diabetes that were not treated with insulin sensitizers, and men with untreated diabetes. Thus, it is unclear whether metformin has positive, negative, or negligible effects on muscle mass and strength in humans. Insulin has anabolic effects on muscle mass and protein metabolism in young and non-insulin resistant men and women; however, this does not seem to occur for individuals with T2DM [39]. Indirectly, insulin therapy may improve muscle health by reducing blood glucose concentrations, which, as described above, can have negative effects on muscle through inflammation, oxidative stress and increased AGEs if chronically elevated. Sulfonylureas have been reported to cause muscle atrophy in a very small proportion of users; however, to the best of our knowledge there are no clinical trials investigating this relationship in a controlled setting. Incretin-based drugs such as DPP-4 inhibitor [39] and liraglutide [40,41] does not seem to decrease muscle. Be careful about sarcopenia by measuring muscle mass etc. by SGLT2 inhibitor administration for liver cirrhosis cases and judge the indication carefully [42]. Overall, there is a paucity of data pertaining to the relationship between glucose-lowering medication and muscle health in humans.

\section{The Association of Antidiabetic Medications with HCC}

DM is an independentrisk factor for HCC in CLD, including chronic hepatitis $\mathrm{C}$ and NAFLD. Exogenous insulin and insulin secretagogues such as sulfonylurea are known to increase the risk of HCC in patients with CLD $[43,44]$. On the other hand, insulin sensitizers such as metformin or thiazolidinediones are reported to reduce HCC risk in patients with CLD [45]. These agents exert antitumor effects by improving hyperinsulinemia as well as activation of AMPK and PPAR $\gamma$. Accumulating evidences of retrospective studies suggest that metformin significantly reduce HCC risk, but two large randomized controlled trials (RCTs), namely, ADOPT (A Diabetes Outcome Progression Trial) and RECORD (Rosiglitazone Evaluated for Cardiovascular Outcomes and Regulation of Glycaemia in Diabetes), failed to demonstrate that metformin offered any chemo preventive effect on the development of HCC when compared with TZDs, but remained potentially advantageous over sulphonyl urea [46]. On the other hand, metformin was recently reported to increase tumor aggressiveness, and resistance to sorafenib caused upregulation of sirtuin-3 $[47,48]$. There is still a lack of both basic and clinical investigations into whether dipeptidyl peptidase 4 (DPP-4) inhibitors have antitumor effects against HCC. CD26, a multifunctional transmembrane glycoprotein, is expressed in HCC and functions DPP-4. Thus, DPP-4 inhibitors exert antitumor effects against HCC in a mice model [49]. DPP-4 inhibitor is reported to upregulate metastatic capacity of HCC by activating nuclear factor E2-related factor 2 to decrease reactive oxygen species levels [50]. 
Harada et al. [51] reported an association between DPP-4 inhibitor and rapid progression of HCC [51]. Thus, further study is required to evaluate beneficial effects of DPP-4 inhibitor on patients with HCC. Influence of GLP-1 RA on hepatocarinogeneis remains unknown. In obese or NASH mice models, SGLT2 inhibitors could prevent the development and growth of HCC [52-55]. In the clinical setting, Kawaguchi and colleagues reported a cirrhotic patient with diabetes mellitus showed spontaneous regression of hepatocellular carcinoma via reduction in angiogenesis-related cytokines [56]. There have no reports preventing hepatocarcinogenesis of SGLT2 inhibitors in T2DM/NAFLD patients. We should select antidiabetic agents from the viewpoint of hepatocarcinogenesis suppression in cirrhotic patients with DM.

\section{Conclusion}

From the above, I would like to propose the management of diabetes that has complicated liver cirrhosis patients (Figure 1). The combination of diabetes in patients with liver cirrhosis obviously worsens the prognosis. On the other hand, it is also pointed out that diabetes may promote the progression of liver fibrosis, and treatment from the viewpoint of breaking the vicious circle of both parties is required. The choice of antidiabetic agents may influence the prognosis of liver disease, and after sufficiently understanding the drug characteristics from among a wide variety of action mechanisms, the selection of a drug that matches the liver condition is sought.

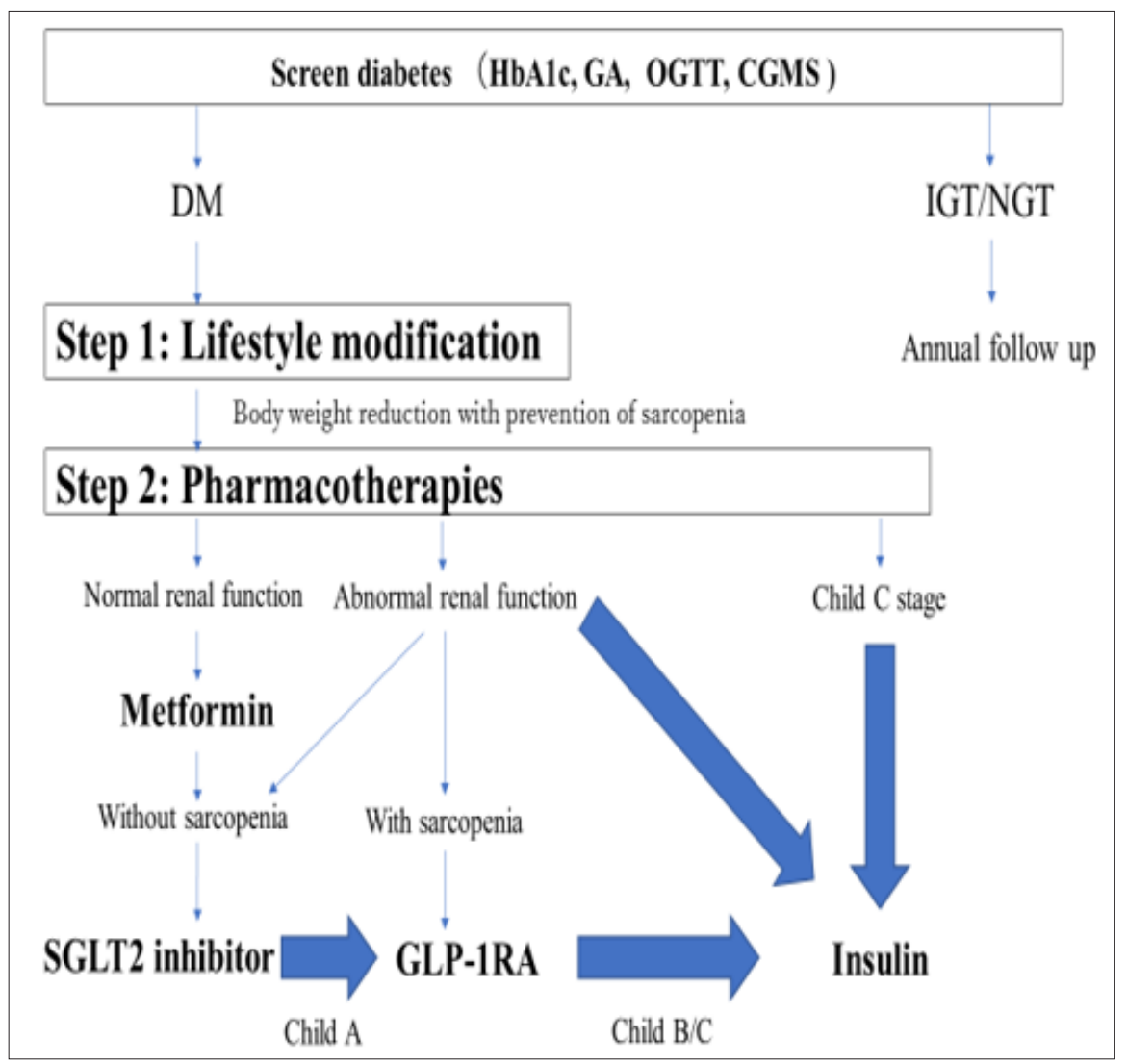

Figure 1: Strategy for diagnosis and treatment in type 2 diabetes with cirrhosis.

HbA1c: Glycated Hemoglobin; GA: Glycated Albumin; OGTT: Oral Glucose Tolerance Test; CGMS: Continuous Glucose Monitoring System; DM: Diabetes Mellitus; IGT: Impaired Glucose Tolerance; NGT: Normal Glucose Tolerance; SGLT2: Sodium-Glucose Cotransporter 2; GLP-1RA: Glucagon Like Peptide-1 Receptor Agonist.

\section{Acknowledgment}

This study was supported by a members of Japan Strategic Medical Administration Research Center (J-SMARC). This research was supported by AMED under Grant Number 19fk0210040h0002.

\section{Conflict of Interest Statement}

Dr. Sumida received honoraria from Mitsubishi Tanabe, Sumitomo Dainippon, Astrazeneka, Ono, and Taisho pharm. Dr.
Sumida received research funding from Bristol-Meyers Squibb. Dr. Nakajima received honoraria from Gilead, Bristol-Meyers Squibb, Novartis, and EA pharma. Dr. Nakajima received research funding from EA pharma, Mylan, and EPD.

\section{References}

1. García Compeán D, González González JA, Lavalle González FJ, González Moreno EI, Maldonado Garza HJ, et al. (2015) The treatment of diabetes 
mellitus of patients with chronic liver disease. Ann Hepatol 14(6): 780788.

2. Koga M, Kasayama S, Kanehara H, Bando Y (2008) CLD (chronic liver diseases)-HbA1C as a suitable indicator for estimation of mean plasma glucose in patients with chronic liver diseases. Diabetes Res Clin Pract 81(2): 258-262.

3. Trenti T, Cristani A, Cioni G, Pentore R, Mussini C, et al. (1990) Fructosamine and glycated hemoglobin as indices of glycemic control in patients with liver cirrhosis. Ric Clin Lab 20(4): 261-267.

4. Suzuki K, Endo R, Kohgo Y, Ohtake T, Ueno Y, et al. (2012) Guidelines on nutritional management in Japanese patients with liver cirrhosis from the perspective of preventing hepatocellular carcinoma. Hepatol Res 42(7): 621-626.

5. Ian J Martins (2015) Nutrition increases survival and reverses NAFLD and Alzheimer's disease. ( $1^{\text {st }}$ edn), In: Alina Berdos (Ed.), Lap Lambert Publishing.

6. Papazafiropoulou A, Andreas Melidonis (2019) Antidiabetic agents in patients with hepatic impairment. World J Meta Anal 7(8): 380-388.

7. Gangopadhyay KK, Singh P (2017) Consensus statement on dose modifications of antidiabetic agents in patients with hepatic impairment. Indian J Endocrinol Metab 21(2): 341-354.

8. Ochi T, Kawaguchi T, Nakahara T, Ono M, Noguchi S, et al. (2017) Differences in characteristics of glucose intolerance between patients with NAFLD and chronic hepatitis C as determined by CGMS. Sci Rep 7(1): 10146.

9. (2018) Diabetes Care 41: S73-S85.

10. Zhang X, Harmsen WS, Mettler TA, Kim WR, Roberts RO, et al. (2014) Continuation of metformin use after a diagnosis of cirrhosis significantly improves survival of patients with diabetes. Hepatology 60(6): 20082016.

11. Loomba R (2014) Rationale for conducting a randomized trial to examine the efficacy of metformin in improving survival in cirrhosis: Pleiotropic effects hypothesis. Hepatology 60(6): 1818-1822.

12. Zhang X, Harmsen WS, Mettler TA, Kim WR, Roberts RO, et al. (2014) Continuation of metformin use after a diagnosis of cirrhosis significantly improves survival of patients with diabetes. Hepatology 60(6): 20082016.

13. Belfort R, Harrison SA, Brown K, Darland C, Finch J, et al. (2006) A placebo controlled trial of pioglitazone in subjects with nonalcoholic steatohepatitis. N Engl J Med 355(22): 2297-2307.

14. Aithal GP, Thomas JA, Kaye PV, Lawson A, Ryder SD, et al. (2008) Randomized, placebo-controlled trial of pioglitazone in nondiabetic subjects with nonalcoholic steatohepatitis. Gastroenterology 135(4): 1176-1184.

15. Cusi K, Orsak B, Bril F, Lomonaco R, Hecht J, et al. (2016) Long-Term pioglitazone treatment for patients with nonalcoholic steatohepatitis and prediabetes or type 2 diabetes mellitus: A randomized trial. Ann Intern Med 165(5): 305-315.

16. Harrison SA, Alkhouri N, Davison BA, Sanyal A, Edwards C, et al. (2019) Insulin sensitizer MSDC-0602K in non-alcoholic steatohepatitis: A randomized, double-blind, placebo-controlled phase IIb study. J Hepatol 19: 30650-30656.

17. Tolman KG, Fonseca V, Dalpiaz A, Tan MH (2007) Spectrum of liver disease in type 2 diabetes and management of patients with diabetes and liver disease. Diabetes Care 30(3): 734-743.

18. Ampuero J, Ranchal I, Nuñez D, Díaz Herrero Mdel M, Maraver M, et al. (2012) Metformin inhibits glutaminase activity and protects against hepatic encephalopathy. PLoS One 7(11): e49279.
19. Gentile S, Guarino G, Romano M, Alagia IA, Fierro M, et al. (2005) A randomized controlled trial of acarbose in hepatic encephalopathy. Clin Gastroenterol Hepatol 3(2): 184-191.

20. Gentile S, Turco S, Guarino G, Oliviero B, Annunziata S, et al. (2001) Effect of treatment with acarbose and insulin in patients with noninsulin-dependent diabetes mellitus associated with non-alcoholic liver cirrhosis. Diabetes Obes Metab 3(1): 33-40.

21. Golightly LK, Drayna CC, McDermott MT (2012) Comparative clinical pharmacokinetics of dipeptidyl peptidase-4 inhibitors. Clin Pharmacokinet 51(8): 501-514.

22. Kaji K, Yoshiji H, Ikenaka Y, Noguchi R, Aihara Y, et al. (2014) Dipeptidyl peptidase-4 inhibitor attenuates hepatic fibrosis via suppression of activated hepatic stellate cell in rats. J Gastroenterol 49(3): 481-491

23. Abrahami D Douros A, Yin H, Yu OHY, Renoux C, et al. (2018) Dipeptidyl peptidase-4 inhibitors and incidence of inflammatory bowel disease among patients with type 2 diabetes: population based cohort study. BMJ 360: k872.

24. Seko Y, Sumida Y, Sasaki K, , Itoh Y, Iijima H, et al. (2018) Effects of canagliflozin, an SGLT2 inhibitor, on hepatic function in Japanese patients with type 2 diabetes mellitus: pooled and subgroup analyses of clinical trials. J Gastroenterol 53(1): 140-151.

25. Sumida Y, Murotani K, Saito M, Tamasawa A, Osonoi Y, et al. (2019) Effect of luseogliflozin on hepatic fat content in type 2 diabetes patients with NAFLD: A prospective, single arm trial (LEAD trial). Hepatol Res 49(1): 64-71.

26. Seko Y, Nishikawa T, Umemura A, Yamaguchi K, Moriguchi M, et al. (2018) Efficacy and safety of canagliflozin in type 2 diabetes mellitus patients with nonalcoholic steatohepatitis and fibrosis. Diabetes, Metabolic Syndrome and Obesity: Targets and Therapy 11: 835-843.

27. Takeda A, Irahara A, Nakano A, Takata E, Koketsu Y, et al. (2017) The improvement of the hepatic histological findings in a patient with nonalcoholic steatohepatitis with type 2 diabetes after the administration of the sodium-glucose cotransporter 2 inhibitor ipragliflozin. Intern Med 56(20): 2739-2744.

28. Saffo S, Taddeui T (2018) SGLT2 inhibitors and cirrhosis: A unique perspective on the comanagement of diabetes mellitus and ascites. Clin Liver Dis 11(6): 141-144.

29. Kaji K, Nishimura N, Seki K, Sato S, Saikawa S, et al. (2018) Sodium glucose cotransporter 2 inhibitor canagliflozin attenuates liver cancer cell growth and angiogenic activity by inhibiting glucose uptake. Int J Cancer 142(8): 1712-1722.

30. Ian J Martins (2018) Insulin therapy and autoimmune disease with relevance to nonalcoholic fatty liver disease. Non Alcoholic Fatty Liver Disease. An Update, Intech Open, Japan. pp. 1-14.

31. Elkrief L, Rautou PE, Sarin S, Valla D, Paradis V, et al. (2016) Diabetes mellitus in patients with cirrhosis: clinical implications and management. Liver Int 36(7): 936-948.

32. Wlazlo N, Beijers HJ, Schoon EJ, Sauerwein HP, Stehouwer CD, et al. (2010) High prevalence of diabetes mellitus in patients with liver cirrhosis. Diabet Med 27(11): 1308-1311.

33. Vial G, Chauvin MA, Bendridi N, Durand A, Meugnier E, et al. (2015) Imeglimin normalizes glucose tolerance and insulin sensitivity and improves mitochondrial function in liver of a high-fat, high-sucrose diet mice model. Diabetes 64(6): 2254-2264.

34. Mudaliar S, Henry RR, Sanyal AJ, Morrow L, Marschall HU, et al. (2013) Efficacy and safety of the farnesoid $\mathrm{X}$ receptor agonist obeticholic acid in patients with type 2 diabetes and nonalcoholic fatty liver disease. Gastroenterology 145(3): 574-582. 
35. Ian James Martins (2018) Sirtuin 1, a diagnostic protein marker and its relevance to chronic disease and therapeutic drug interventions. EC Pharmacology and Toxicology 6(4): 209-215.

36. Ebadi M, Bhanji RA, Mazurak VC, Montano Loza AJ (2019) Sarcopenia in cirrhosis: from pathogenesis to interventions. J Gastroenterol 54(10): 845-859.

37. Cetrone M, Mele A, Tricarico D (2014) Effects of the antidiabetic drugs on the age-related atrophy and sarcopenia associated with diabetes type II. Curr Diabetes Rev 10(4): 231-237.

38. Mesinovic J, Zengin A, De Courten B, Ebeling PR, Scott D (2019) Sarcopenia and type 2 diabetes mellitus: A bidirectional relationship. Diabetes Metab Syndr Obes 12: 1057-1072.

39. Bouchi R, Fukuda T, Takeuchi T, Nakano Y, Murakami M, et al. (2018) Dipeptidyl peptidase 4 inhibitors attenuates the decline of skeletal muscle mass in patients with type 2 diabetes. Diabetes Metab Res Rev $34(2)$.

40. Perna S, Guido D, Bologna C, Solerte SB, Guerriero F, et al. (2016) Liraglutide and obesity in elderly: efficacy in fat loss and safety in order to prevent sarcopenia. A perspective case series study. Aging Clin Exp Res 28(6): 1251-1257.

41. Ishii S, Nagai Y, Sada Y, Fukuda H, Nakamura Y, et al. (2019) liraglutide reduces visceral and intrahepatic fat without significant loss of muscle mass in obese patients with type 2 diabetes: A prospective case series. J Clin Med Res 11(3): 219-224.

42. Sasaki T, Sugawara M, Fukuda M (2019) Sodium-glucose cotransporter 2 inhibitor-induced changes in body composition and simultaneous changes in metabolic profile: 52-week prospective LIGHT (Luseogliflozin: the Components of Weight Loss in Japanese Patients with Type 2 Diabetes Mellitus) Study. J Diabetes Investig 10(1): 108-117.

43. Singh S, Singh PP, Singh AG, Murad MH, Sanchez W (2013) Anti-diabetic medications and the risk of hepatocellular cancer: A systematic review and meta-analysis. Am J Gastroenterol 108(6): 881-891.

44. Kawaguchi T, Taniguchi E, Morita Y, Shirachi M, Tateishi I, et al. (2010) Association of exogenous insulin or sulphonylurea treatment with an increased incidence of hepatoma in patients with hepatitis $C$ virus infection. Liver Int 30(3): 479-486.

45. Decensi A, Puntoni M, Goodwin P, Cazzaniga M, Gennari A, et al. (2010) Metformin and cancer risk in diabetic patients: A systematic review and meta-analysis. Cancer Prev Res (Phila) 3(11): 1451-1461.

46. Home PD, Kahn SE, Jones NP, Noronha D, Beck Nielsen H, et al. (2010) Experience of malignancies with oral glucose-lowering drugs in the randomised controlled ADOPT (A diabetes outcome progression trial) and RECORD (rosiglitazone evaluated for cardiovascular outcomes and regulation of glycaemia in diabetes) clinical trials. Diabetologia 53(9): 1838-1845.

47. Casadei Gardini A, Faloppi L, De Matteis S, Foschi FG, Silvestris N, et al. (2017) Metformin and insulin impact on clinical outcome in patients with advanced hepatocellular carcinoma receiving sorafenib: Validation study and biological rationale. Eur J Cancer 86: 106-114.

48. Casadei Gardini A, Marisi G, Scarpi E, Scartozzi M, Faloppi L, et al. (2015) Effects of metformin on clinical outcome in diabetic patients with advanced HCC receiving sorafenib. Expert Opin Pharmacother 16(18): 2719-2725.

49. Sohji Nishina, Akira Yamauchi, Takumi Kawaguchi, Kohei Kaku, Moritaka Goto, et al. (2019) Dipeptidyl peptidase 4 inhibitors reduce hepatocellular carcinoma by activating lymphocyte chemotaxis in mice. Cell Mol Gastroenterol Hepatol 7(1): 115-134.

50. Wang H, Liu X, Long M, Huang Y, Zhang L, et al. (2016) NRF2 activation by antioxidant antidiabetic agents accelerates tumor metastasis. Sci Transl Med 8(334): 334-351.

51. Harada M, Yoneda A, Haruyama S, Yabuki K, Honma Y, et al. (2017) Bullous pemphigoid associated with the dipeptidyl peptidase-4 inhibitor sitagliptin in a patient with liver cirrhosis complicated with rapidly progressive hepatocellular carcinoma. Intern Med 56(18): 2471-2474.

52. Obara K, Shirakami Y, Maruta A, Ideta T, Miyazaki T et al. (2017) Preventive effects of the sodium glucose cotransporter 2 inhibitor tofogliflozin on diethylnitrosamine-induced liver tumorigenesis in obese and diabetic mice. Oncotarget 8(35): 58353-58363.

53. Kaji K, Nishimura N, Seki K, Sato S, Saikawa S, et al. (2018) Sodium glucose cotransporter 2 inhibitor canagliflozin attenuates liver cancer cell growth and angiogenic activity by inhibiting glucose uptake. Int J Cancer 142(8): 1712-1722.

54. Shiba K, Tsuchiya K, Komiya C, Miyachi Y, Mori K, et al. (2018) Canagliflozin, an SGLT2 inhibitor, attenuates the development of hepatocellular carcinoma in a mouse model of human NASH. Sci Rep 8(1): 2362.

55. Hung MH, Chen YL, Chen LJ, Chu PY, Hsieh FS, et al. (2019) Canagliflozin inhibits growth of hepatocellular carcinoma via blocking glucose-influxinduced $\beta$-catenin activation. Cell Death Dis 10(6): 420.

56. Kawaguchi T, Nakano D, Okamura S, Shimose S, Hayakawa M, et al. (2019) Spontaneous regression of hepatocellular carcinoma with reduction in angiogenesis-related cytokines after treatment with sodium-glucose cotransporter 2 inhibitor in a cirrhotic patient with diabetes mellitus. Hepatol Res 49(4): 479-486. 